\title{
Tercerventriculo-cisternostomía microquirúrgica. Experiencia personal y descripción de acceso micropterional como alternativa a acceso keyhole supraorbitario
}

\section{Microsurgical third ventricle-cisternostomy. Personal experience and micropterional technique description as an alternative for the supraorbital keyhole approach}

\section{Luis Contreras Seitz}

${ }^{1}$ Neurocirujano, Hospital Clínico Universidad de Chile, Clínica Las Condes. Santiago, Chile.

Presentado en el LXI Congreso Chileno de Neurocirugía como trabajo de ingreso a la sociedad chilena de neurocirugía. Puerto Varas. Octubre de 2018.

Recibido: 17/12/18; Aceptado: 28/12/18

\begin{abstract}
Resumen
La hidrocefalia es una patología frecuente en el tabajo del neurocirujano. Puede clasificarse en comunicante o no comunicante según sea su etiología. La cirugía derivativa ventrículo-peritoneal es el estándar para el manejo de esta patología, sin embargo, esta asociada a complicaciones como la obstrucción o infección. Para la hidrocefalia no comunicante u obstructiva existe la tercer-ventrículo cisternostomía (TVC) como altetrnativa para su tratamiento. Esta técnica se realiza de forma endoscopica con buenos resultados y bajas complicaciones. En pacientes con hidrocefalia obstructiva en donde no se puede realizar una endoscopía por características anatómicas del paciente, existen reportes de realizar este procedimiento mediante una técnica microquirúrgica, clasicamente por vía supraorbitaria keyhole. Entre los años 2015 y 2018 se realizaron en 17 pacientes con hidrocefalia obstructiva una TVC microquirúrgica con un $82 \%$ de éxito y sin complicaciones postoperatorias atribuibles a la cirugía. El $82 \%$ se realizó mediante un acceso micropterional (que se describe en este artículo), 12\% mediante keyhole supraorbitario y $6 \%$ mediante minipterional. La TVC microquirúrgica podría ser considerada una alternativa para el manejo de la hidrocefalia obstructiva en casos donde no se pueda realizar la endoscopia. La tasa de efectividad de la presente serie es comparable a la de la TVC endoscópica. Más trabajos se deben realizar para comparar la técnica vía acceso micropterional versus keyhole supraorbitario.
\end{abstract}

Palabras clave: Hidrocefalia, ventriculocisternostomia.

\begin{abstract}
\section{Correspondencia a:}

Luis Contreras Seitz

Santos Dumont 999, Independencia. Santiago, Chile.

Telefono: +56 229788260

luis.contreras.seitz@uchile.cl
\end{abstract}

Hydrocephalus is a frequent pathology in a neurosurgeon work. It can be classified as communicating or non-communicating according to its etiology. Ventriculoperitoneal shunt is the standard treatment for this pathology, however, it is associated with complications such as obstruction and/or infection. For non-communicating (or obstructive) hydrocephalus, the thirdventricle cisternostomy (TVC) is an alternative for its treatment. This technique is performed endoscopically with good results and low complications. In patients with obstructive hydrocephalus where endoscopy cannot be performed due to 
anatomical characteristics of the patient, there are reports of performing this procedure using a microsurgical technique, classically via a supraorbital keyhole approach. Between 2015 and 2018, a microsurgical TVC was performed in 17 patients with obstructive hydrocephalus. The technique had a $82 \%$ success rate and no postoperative complications attributable to surgery. The $82 \%$ was performed through a micropterional approach (described in this article), $12 \%$ through supraorbital keyhole and $6 \%$ through a mini-pterional. The microsurgical TVC could be considered an alternative for the management of obstructive hydrocephalus in cases where endoscopy can not be performed. The effectiveness rate of the present series is comparable to that of endoscopic TVC. More publications are needed to compare the micropterional versus the supraorbital keyhole approach benefits.

Key words: Hydrocephalus, ventriculocisternostomy.

\section{Introducción}

La hidrocefalia se clasifica clásicamente como comunicante o no comunicante $u$ obstructiva dependiendo de su etiología. La primera se produce principalmente con un trastorno de la reabsorción del líquido cefalorraquídeo (LCR) y la segunda por causa de alguna obstrucción en algún punto de la anatomía ventricular que impide la correcta circulación del LCR con aumento de la presión hidrostática de manera retrograda.

El tratamiento más aceptado y frecuente de la hidrocefalia es la derivación del LCR desde los ventrículos a la cavidad peritoneal mediante una válvula derivativa ventrículo-peritoneal (DVP) ${ }^{1}$. Dada la alta tasa de disfunción y revisión de estos sistemas, nuevas alternativas han aparecido. Es así como la tercer-ventriculocisternostomía se ha establecido como alternativa a la DVP principalmente en pacientes con estenosis del acueducto de Silvio ${ }^{2,3}$.

Los pacientes con hidrocefalia obstructiva, principalmente estenosis del acueducto de Silvio, pueden ser tratados con altas tasas de éxito mediante una tercer-ventriculocisternostomía, que consiste en comunicar el espacio ventricular con el cisternal y de esa manera "crear" una nueva ruta al LCR y resolver la hidrocefalia ${ }^{3}$. La mayor experiencia en esta técnica es mediante la técnica endoscópica, siendo reportados también series microquirúrgicas con buenos resultados ${ }^{4,5,6,7}$.

La técnica endoscópica consiste en la navegación ventricular a través de un trepano frontal, entrar al tercer ventrículo a través del foramen de monro y abrir el piso del tercer ventrículo para comunicar el ventrículo con la cisterna interpeduncular. El paciente ideal debe presentar un sistema ventricular amplio con un foramen de monro dilatado y una cisterna interpeduncular amplia con una bifurcación basilar idealmente alejada del piso del tercer ventrículo. La tercerventriculocisternostomía endoscópica ha demostrado buenos resultados en casos de hidrocefalia obstructiva como terapia de primera línea. Sus complicaciones bajas, siendo principalmente la hemorragia (hemorragia leve a ruptura de arteria basilar y muerte) y daño neural (daño de fornix, hipotálamo y mesencéfalo). La morbilidad descrita es de un $8 \%$ con una mortalidad del 0,29\%,3,8.

La técnica microquirúrgica mediante un acceso keyhole supraorbitario ha sido descrita como alternativa a la técnica endoscópica para casos donde la técnica endoscópica pudiera ser más riesgosa o complicada como lo son pacientes con ventrículos pequeños o con una bifurcación basilar muy alta en contacto con el piso del tercer ventrículo. Si bien, las series publicadas son más pequeñas que las series de endoscopía, su efectividad es alta con bajo riesgo de complicaciones, siendo la principal la fístula de LCR por apertura del seno frontal y la estética por la cicatriz ciliar propia del acceso. La principal ventaja de la técnica microquirúrgica es el mayor control de las posibles hemorragias que pudieran ocurrir y el trabajo por espacios cisternales sin dañar tejido neural ${ }^{9,10,11}$.

En orden de disminuir las complicaciones de la técnica microquirúrgica por un acceso keyhole supraorbitario, mediante una variación del acceso minipterional, en el presente trabajo se describe un acceso keyhole más lateral o "micropterional", donde la probabilidad de apertura del seno frontal es nula y donde el defecto estético es mínimo al realizar la incisión tras la línea de inserción del cabello y quedando el acceso totalmente cubierto por el músculo temporal superficial. A continuación, se describe la experiencia personal del autor en tercerventriculo-cisternostomía microquirúrgica y la descripción de la técnica quirúrgica mediante un acceso micropterional como variante al acceso keyhole supraorbitario.

\section{Materiales y Métodos}

Estudio prospectivo descriptivo de casos operados de tercerventrículo-cisternostomía microquirúrgica por el autor entre los años 2014 y 2018.

Técnica quirúrgica de tercerventrículo-cisternostomía vía acceso micropterional (Figura 1).

Paciente es posicionado en decúbito supino con cabeza fija en cabezal de mayfield. La cabeza es rotada en $45^{\circ}$ contrario a acceso quirúrgico con hiperextensión cervical. Se realiza rasurado lineal en zona de incisión cutánea. Se realiza aseo quirúrgico y se instalan los paños quirúrgicos en forma habitual.

Se realiza una incisión cutánea por detrás de la línea de inserción del pelo desde el arco cigomático hasta el punto medio entre la proyección de la pared lateral de la órbita y la línea media pupilar (Figura 1A). Se realiza una incisión en la fascia temporal superficial para hacer un colgajo interfascial con el fin de proteger la rama frontal del nervio facial (Figura 1B). Se visualiza la línea temporal superior donde se implanta músculo temporal superficial y apófisis frontal de cigoma.

Se realiza sección y disección del músculo temporal 
Figura 1. Descripción de técnica de tercerventriculocisternostomía microquirúrgica vía micropterional.
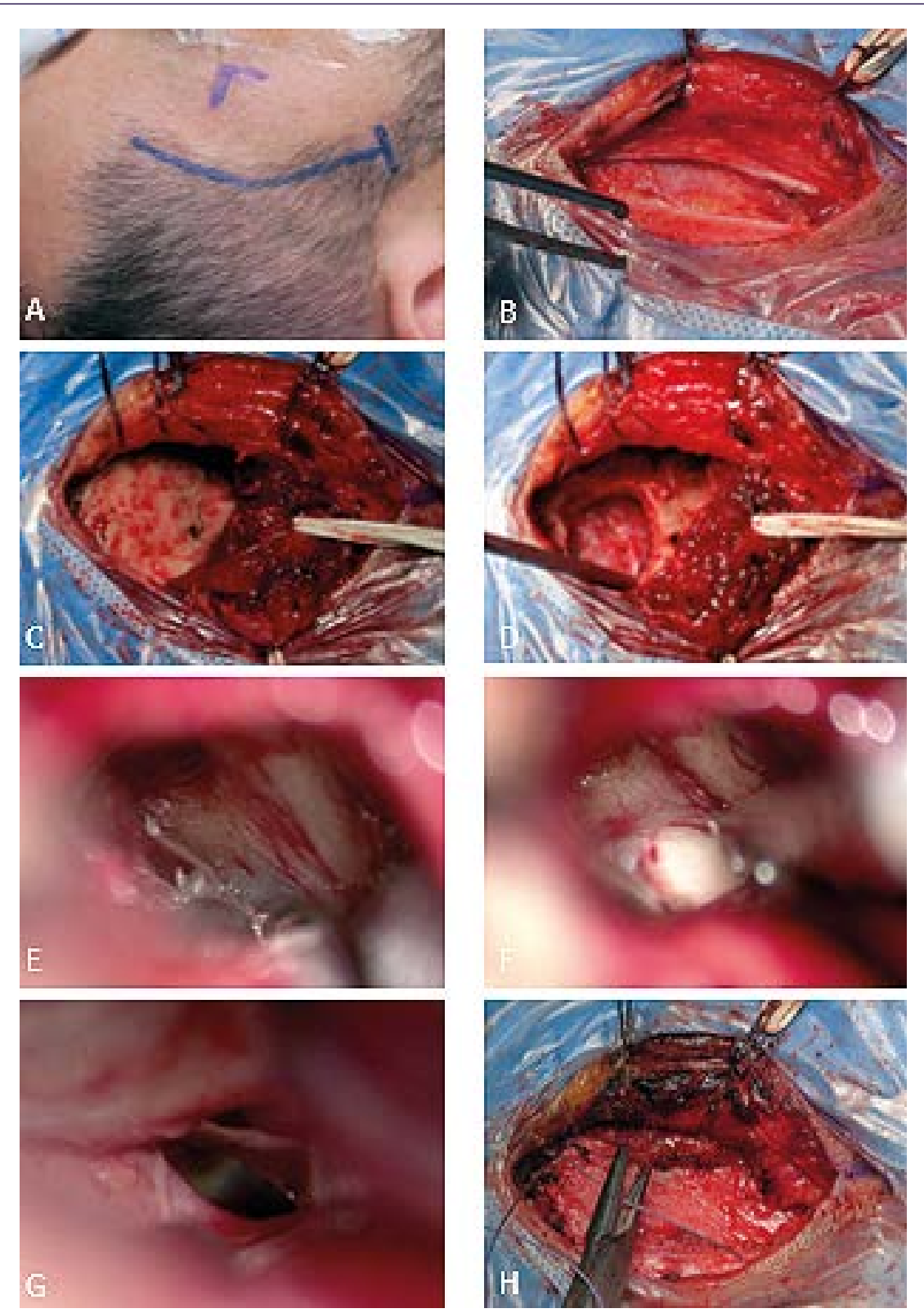

superficial hacia posterior desinsertándolo de línea temporal superior y apófisis frontal de cigoma (Figura 1C).

Se identifica el keyhole de Mccarthy y se realiza pequeña craneotomía de $2 \mathrm{~cm}$ aproximadamente con borde superior en línea temporal superior, y borde inferior en la porción superior del ala esfenoidal y hacia anterior lo más basal posible (Figura 1D).

Se realiza hemostasia prolija para prevenir sangrado extradural. Si es necesario se puede rebajar con motor borde superior del ala esfenoidal.

Bajo microscopio se realiza apertura arciforme de duramadre con base antero inferior.

Con paciencia se realiza disección subfrontal siguiendo ala esfenoidal hasta identificar el nervio óptico. Se abre cisterna óptico-carotídea, la apertura de esta cisterna permite salida de líquido cefalorraquídeo que facilita la disección. Se sigue nervio óptico hasta encontrar quiasma y lámina terminalis (Figura 1E).

Una vez identificada la lámina terminalis se abre de manera amplia coagulando posteriormente los bordes de su apertura (Figura 1F). Una vez abierta se procede a abrir membrana de liliequist por espacio óptico-carotideo o lateral a arteria carótida interna supraclinoidea (Figura 1G).

Se realiza hemostasia prolija sin dejar ningún punto sangrante, para posteriormente realizar un cierre hermético de duramadre con sutura. Se aplica sellante dural sobre durorrafia y se repone hueso con placas material de fijación. También se puede realizar craneoplastía con malla de titanio o acrílico.

Posteriormente, se vuelve a implantar músculo temporal 


\begin{tabular}{|c|c|c|c|c|}
\hline \multicolumn{5}{|c|}{$\begin{array}{l}\text { Tabla 1. Descripción de pacientes sometidos a TVC microquirúrgica } \\
\text { lista de pacientes sometidos a tercerventrículo-cisternostomía microquirúrgica }\end{array}$} \\
\hline & Edad/Sexo & Diagnóstico primario & Acceso & DVP posterior \\
\hline 1 & $26 / \mathrm{M}$ & Tumor pineal & Keyhole supraorbitario & No \\
\hline 2 & 9/F & Glioma de tronco & Minipterional & No \\
\hline 3 & $58 / \mathrm{M}$ & $\begin{array}{l}\text { Estenosis acueducto de Silvio/hidrocefalia } \\
\text { normotensiva }\end{array}$ & Micropterional & $\mathrm{Si}$ \\
\hline 4 & $69 / \mathrm{M}$ & $\begin{array}{l}\text { Estenosis acueducto de Silvio/hidrocefalia } \\
\text { normotensiva }\end{array}$ & Micropterional & No \\
\hline 5 & $27 / F$ & $\begin{array}{l}\text { Tumor } 4 \text { to ventrículo operado, hemoventrículo } \\
\text { postoperatorio }\end{array}$ & Micropterional & No \\
\hline 6 & $77 / \mathrm{M}$ & HIC cerebelosa/hidrocafalia & Micropterional & No \\
\hline 7 & $55 / F$ & Carcinomatosis meningea/estenosis acueducto & Micropterional & DVE \\
\hline 8 & $68 / \mathrm{M}$ & Fístula AV rota/hemoventrículo & Micropterional & No \\
\hline 9 & $51 / \mathrm{M}$ & $\begin{array}{l}\text { Estenosis acueducto de Silvio/hidrocefalia } \\
\text { normotensiva }\end{array}$ & Micropterional & No \\
\hline 10 & $32 / \mathrm{M}$ & Tumor 4 to ventrículo operado & Micropterional & No \\
\hline 11 & $39 / F$ & Cavernoma mescencéfalo & Micropterional & No \\
\hline 12 & $63 / \mathrm{M}$ & Glioblastoma de la región pineal/siembra meningea & Micropterional & $\mathrm{Si}$ \\
\hline 13 & $16 / \mathrm{M}$ & Estenosis acueducto de Silvio & Micropterional & No \\
\hline 14 & $67 / \mathrm{M}$ & Estenosis acueducto de Silvio & Keyhole supraorbitario & No \\
\hline 15 & $33 / \mathrm{M}$ & Tumor pineal & Micropterional & No \\
\hline 16 & $63 / \mathrm{M}$ & Meningioma falcotentorial gigante & Micropterional & No \\
\hline 17 & $49 / \mathrm{M}$ & Hemorragia talámica & Micropterional & No \\
\hline
\end{tabular}

superficial anclándolo a línea temporal superior y apófisis frontal de cigoma con suturas ocultando el defecto óseo bajo el musculo (Figura $1 \mathrm{H}$ ).

Posteriormente, se procede a cierre de gálea en forma habitual y de piel con sutura intradérmica.

\section{Resultados}

Se realizó una tercerventrículocisternostomía microquirúrgica en 17 pacientes con hidrocefalia (Tabla 1). Un $82 \%$ fue realizadas por vía microptreional, $6 \%$ por vía minipterional y $12 \%$ por vía keyhole supraorbitario. El procedimiento tuvo un éxito de un $82 \%$, siendo necesario en un $18 \%$ la instalación de una DVP o DVE. Un paciente $(5,8 \%)$ falleció 4 días posterior al procedimiento por causa no relacionada a la cirugía. No hubo fístula de LCR o infecciones de herida operatoria. El tiempo operatorio promedio fue de $121 \mathrm{~min}$ y para la técnica vía abordaje micropterional de $120 \mathrm{~min}$. La causa de la hidrocefalia fue en un $47 \%$ estenosis del acueducto de Silvio, $29 \%$ tumoral y $24 \%$ hemorrágico. El seguimiento de los pacientes varía entre 41 y 3 meses.

\section{Caso clínico 1}

Paciente de 9 años, sexo femenino, con cuadro de 1 mes de evolución de cefalea y mareos a lo que se agrega diplopía. Al examen la paciente presenta paresia del sexto nervio derecho, parálisis facial central derecha y síndrome piramidal bilateral. Estudio con RM de cerebro muestra lesión expansiva intra-axial a nivel del tronco y puente con dilatación del tercer ventrículo y ventrículos laterales (Figura $2 \mathrm{~A}$ y B). Se decide realizar tercerventrículocisternostomía por craneotomía minipterional, la que se realiza sin incidentes. La paciente mejora su cuadro de cefalea y diplopía con reducción de la talla ventricular (Figura $2 \mathrm{C}$ ). La paciente persiste libre de hidrocefalia hasta que fallece 1 año posterior al diagnóstico.

\section{Caso clínico 2}

Paciente mujer de 28 años que por cuadro de cefalea holocranea progresiva se diagnostica tumor del cuarto ventrículo e hidrocefalia asociada. Paciente evaluada en otro centro donde se instala válvula derivativa ventrículo peritoneal y luego se realiza resección de tumor del cuarto ventrículo. Biopsia informa papiloma de plexo coroide. Imágenes postoperatorias muestran hematoma que ocupa todo el cuarto ventrículo.

La paciente es traslada al Hospital Clínico de la Universidad de Chile a rehabilitación en fisiatría donde ingresa con cuadro de cuadro febril y de disfunción valvular. Se diagnostica cuadro de ventriculitis, por lo que se retira derivativa ventrículo peritoneal, se instala drenaje ventricular externo y se completa tratamiento por 21 días.

Se intenta retiro de drenaje ventricular externo lo cual no es posible dado aumento de talla ventricular y aparición de 


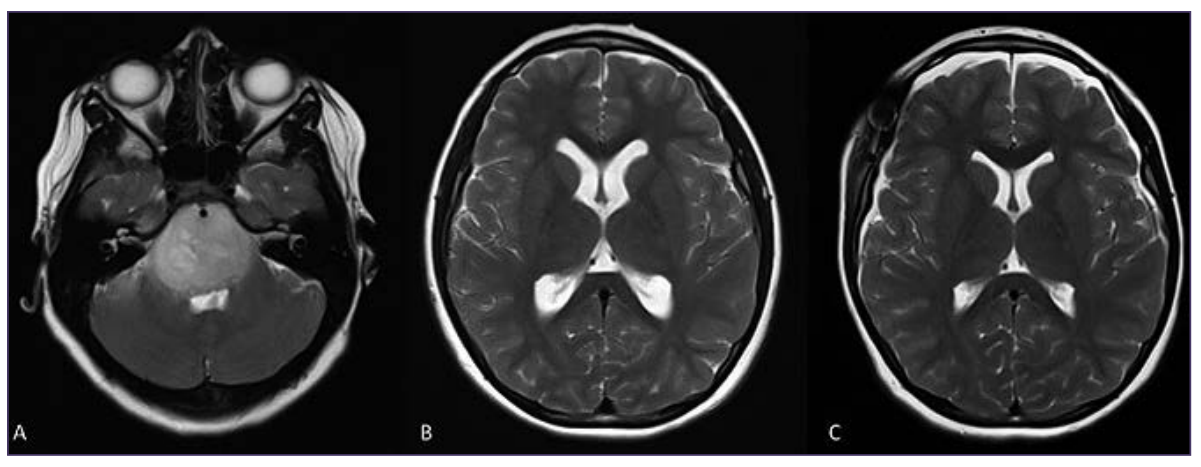

Figura 2. A: Lesión difusa pontina intra-axial sugerente de glioma pontino. B: Dilatacion de sistema ventricular. Resultado por TVC microquirúrgica.

síntomas al cierre de este, junto a esto persiste hematoma del cuarto ventrículo. Se decide realizar tercerventrículocisternostomía microquirúrgica la que se realiza sin incidentes, retirando DVE dos días posterior a la intervención quirúrgica.

Posterior a esto la paciente continua favorablemente su rehabilitación siendo dada de alta sin presentar recurrencia de hidrocefalia durante su seguimiento.

\section{Caso clínico 3}

Paciente de 63 años con antecedentes de HTA y DM2 ingresa por cuadro de cefalea progresiva y síndrome de parinaud. TAC cerebral y RM de cerebro muestran proceso expansivo de la región pineal y lamina cuadrigemina con componentes necróticos y quísticos asociados a hidrocefalia triventricular. Se realiza tercerventrículo-cisternostomía microquirúrgica sin incidentes aliviando cefalea y trastorno de oculomotilidad. Se completa estudio con marcadores tumorales en LCR y suero, los cuales resultan negativos, destacando aumento de proteínas en LCR. Se realiza RM de columna total que muestra múltiples implantes meníngeos. Se realiza biopsia de uno de estos implantes (T10-T11), cuya biopsia informa Glioblastoma IDH no mutado. El paciente es dado de alta reingresando a los 10 días por presentar nuevamente alteración de oculomotilidad a mirada superior y cefalea. Se realiza RM de cerebro que muestra aumento de lesión tumoral pineal, aparición de focos de captación leptomeningea cerebral y aumento de talla ventricular siendo necesaria instalación de DVP con lo que el paciente resuelve sus síntomas y es dado de alta.

El paciente reingresa a los 30 días con compromiso de conciencia (GCS 10) y aumento importante de lesión pineal con componente hemorrágico y compresión de mesencéfalo. El paciente mejora parcialmente con corticoides siendo trasladado para radioterapia y terapia paliativa.

\section{Caso clínico 4}

Paciente de sexo masculino 68 años, ingresa por cuadro de cefalea ictal de 5 días de evolución. TAC de cerebro demuestra hematoma temporal izquierdo y vaciamiento a sistema ventricular con ocupación de cuerno occipital y frontal de ventrículo lateral izquierdo. Ingresa sin déficit neurológico. Se realiza angiografía que muestra fístula arterio venosa dural temporal izquierda la que se emboliza. Posterior a embolización, el paciente se compromete de conciencia. TAC

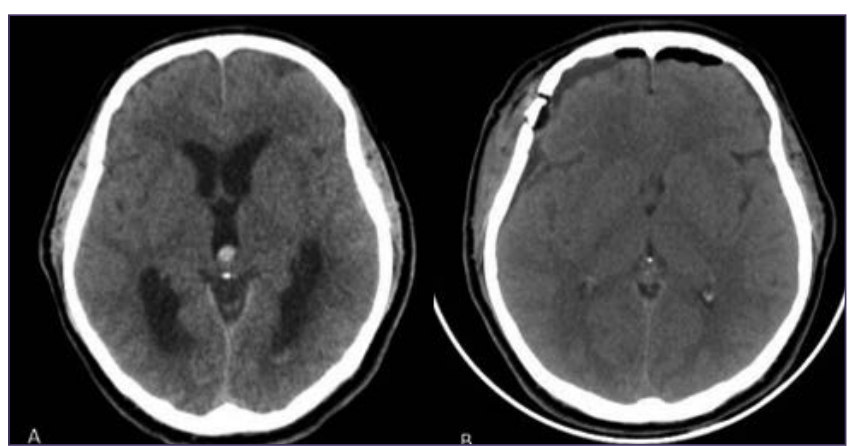

Figura 3. A: Tomografía computada de cerebro sin contraste que muestra hemorragia en tercer ventrículo asociada a dilatación ventricular. B: Resolución de hidrocefalia tras TVC microquirúrgica

cerebral muestra coagulo obstruyendo acueducto de Silvio con dilatación de tercer ventrículo y ventrículos laterales (Figura 3A). Se realiza tercerventriculo-cisternostomía microquirúrgica sin incidentes. TAC de control muestra resolución de hidrocefalia (Figura 3B). Paciente dado de alta sin déficit y $\sin$ hidrocefalia.

\section{Discusión}

El tratamiento de la hidrocefalia es un desafío para el neurocirujano. El standard es la instalación de una válvula derivativa ventrículo peritoneal, sin embargo, implica una alta tasa de disfunción por obstrucción o infección'1. En un subgrupo de pacientes con hidrocefalia obstructiva es posible solucionar esta patología mediante una tercerventrículo-cisternostomía con alta tasa de éxito y bajas complicaciones ${ }^{2,3}$. La principal técnica para la realización de este procedimiento es la endoscopía ${ }^{2,3}$, sin embargo, es necesario contar con la tecnología y que el paciente cumpla con condiciones anatómicas favorables. Una alternativa descrita es la tercerventrículo-cisternostomía microquirúrgica, en la cual se realiza la apertura de la lámina terminalis con o sin apertura de la membrana de liliequist para resolver la hidrocefalia. Esta técnica ha sido descrita por vía keyhole supraorbitario, siendo una limitante de esta técnica, el potencial riesgo de fístula en pacientes con seno frontal prominente y las complicaciones estéticas 
propias del acceso ${ }^{4,5,6,7}$.

En este trabajo se muestra una alternativa de tercerventriculocisternostomía microquirúrgica por vía keyhole micropterionall, donde no hay riesgo de apertura del seno frontal, con menor riesgo teórico de fistula y con mejoras estéticas en pacientes seleccionados, dado que la craneotomía queda bajo el músculo temporal superficial y la incisión cutánea tras la línea de implantación del pelo.

En esta serie se demostró una efectividad de la técnica en un $82 \%$. Los casos en los que fallo se debieron a la fisiopatología de la enfermedad tratada, con un trastorno de reabsorción de LCR asociado a un patrón obstructivo secundario a diseminación tumoral leptomeningea (casos 7 y 12 de Tabla 1) y a subestimar un estudio de flujo de LCR que mostraba una probable comunicación ya existente entre el tercer ventrículo y el espacio cisternal (caso 3, Tabla 1).

\section{Conclusiones}

La tercerventrículo-cisternostomía puede ser considerado dentro de la terapia inicial de la hidrocefalia obstructiva por obstrucción del acueducto de Silvio o del cuarto ventrículo. La técnica microquirúrgica puede ser considerada una alternativa a la técnica endoscópica en casos donde esta ultima no es factible de realizar.

El acceso micro-pterional podría ser considerada una alternativa al acceso keyhole supraorbitario en el tratamiento microquirúrgico de la hidrocefalia obstructiva, sin embargo, son necesarios estudios comparativos para evaluar las ventajas y desventajas con respecto al acceso keyhole supraorbitario y a la técnica endoscópica.

Agradecimientos: a Ivone Lara Vásquez por su paciencia y apoyo.

\section{Referencias}

1. Reddy G, Bollam P. and Caldito G. (2014). Lont-Term Outcomes of ventriculoperitoeal shunt surgery in patients with hydrocephalus. World Neurosurgery, 81(2), pp 404-410.

2. Gliemroth J, Käsbeck E. and Kehler U. (2014). Ventriculocisternostomy versus ventriculoperitoneal shunt in the treatment of hydrocephalus: A retrospective, long-term observational study. Clinical Neurology and Neurosurgery, 122, pp.92-96.

3. Schroeder HW, Oertel J, Gaab MR. Endoscopic treatment of cerebrospinal fluid pathway obstructions. Neurosurgery. 2007;60(2 Suppl 1):ONS44-51.

4. Mura J, Figueiredo EG, Carmona P, Palma-fellemberg Á, De faria JW. The anterior ventriculo-cisternostomy: the pioneers' work revisited. J Neurol Surg A Cent Eur Neurosurg. 2013;74(3):14651.

5. Carmona R. Abordaje minimamente invasivo supraorbitario lateral key-hole: experiencia de 64 casos. Rev. Chil. Neurocirugía. 2011;36: 11-19.

6. Van lindert EJ. Microsurgical third ventriculocisternostomy as an alternative to ETV: report of two cases. Childs Nerv Syst. 2008;24(6):757-61.

7. Meybodi AT, Miri SM. Microscopic supraciliary approach for terminal laminotomy for treatment of hydrocephalus: a preliminary report of eight cases. Turk Neurosurg. 2012;22(5):599-603.

8. Bouras T, Sgouros S. Complications of endoscopic third ventriculostomy: a systematic review. Acta Neurochir Suppl. 2012;113:149-53.

9. Reisch R, Perneczky A, Filippi R. Surgical technique of the supraorbital key-hole craniotomy. Surg Neurol. 2003;59(3):223-7.

10. Reisch R, Perneczky A. Ten-year experience with the supraorbital subfrontal approach through an eyebrow skin incision. Neurosurgery. 2005;57(4 Suppl):242-55.

11. Jallo Gl, Bognár L. Eyebrow surgery: the supraciliary craniotomy: technical note. Neurosurgery. 2006;59(1 Suppl 1):ONSE157-8 